\title{
An Unusual Cause of Diarrhea
}

\author{
Andrew Joelson ${ }^{1}$ (C) Benjamin Lebwohl ${ }^{1} \cdot$ Stuart Bentley-Hibbert ${ }^{2} \cdot$ Steven Lee-Kong ${ }^{3} \cdot$ Oscar Lebwohl $^{1}$
}

Received: 5 January 2021 / Accepted: 19 February 2021 / Published online: 8 March 2021

(c) The Author(s), under exclusive licence to Springer Science+Business Media, LLC part of Springer Nature 2021

Keywords Diarrhea $\cdot$ Surgical complication $\cdot$ Enterocolic fistula $\cdot$ Hernia mesh

The patient is an 83-year-old man with a history of hypertension, inguinal hernia status post laparoscopic mesh repair (1997), prostate cancer status post radical prostatectomy (2012) and radiation therapy, sigmoid diverticulitis status post-sigmoid resection (2017) who presented with several months of non-bloody diarrhea, up to 10 times per day.

In additional to diarrhea, the patient reported generalized fatigue and weakness and had lost $10 \mathrm{lb}$. He was recently initiated on methotrexate for a new diagnosis of rheumatoid arthritis. Physical examination was conducted via video visit due to the coronavirus pandemic and the patient was well appearing at that time. A complete metabolic panel and complete blood count revealed a mild anemia, with hemoglobin of $11.1 \mathrm{~g} / \mathrm{dL}$ and a normal mean corpuscular volume, without leukocytosis. Celiac serologies checked seven months prior were within normal limits and Vitamin B12 at the time was normal. Sedimentation rate was elevated at $46 \mathrm{~mm} / \mathrm{h}$, which was attributed to rheumatoid arthritis. Stool tests for Clostridioides difficile and multiplex gastrointestinal pathogen PCR were negative. The patient was initiated on loperamide as needed and his methotrexate was discontinued at the direction of his rheumatologist. Despite a modest improvement, the patient continued to have watery bowel movements up to five times per day prompting endoscopic evaluation.

His upper endoscopy, including biopsies of the stomach and duodenum was without significant abnormality.

Andrew Joelson

AMJ2181@cumc.columbia.edu

1 Division of Digestive and Liver Disease, Department of Medicine, Columbia University Irving Medical Center, 622 West 168th Street - Room 301, Box 4-303, New York, NY 10032, USA

2 Division of Abdominal Imaging, Department of Radiology, Columbia University Irving Medical Center, New York, USA

3 Division of Colorectal Surgery, Department of Surgery, Columbia University Irving Medical Center, New York, NY, USA
Colonoscopy revealed a patent colorectal anastomosis; however, $10 \mathrm{~cm}$ proximal to the anastomosis, a large piece of intraluminal foreign material with a mesh pattern was noted. Gentle traction failed to dislodge the mesh and induced mild bleeding. The patient was referred for surgical evaluation. A CT scan of the abdomen and pelvis showed erosion of the previously placed left inguinal hernia mesh into the descending colon. The patient underwent exploratory laparoscopy, where he was found to have densely adherent bowel as a result of his prior surgeries. Evaluation of the small and large intestine revealed an enterocolic fistula between the proximal jejunum and the descending colon involving a portion of polypropylene surgical mesh from the left abdominal wall that had eroded into and was identified within both proximal and distal portions of the enterocolic fistula. A portion of proximal jejunum was removed and a primary anastomosis was created. The remainder of the low anterior resection was completed. The patient's postoperative course was complicated by an acute kidney injury and anemia that responded to red blood cell transfusion and he was discharged on postoperative day 7. On follow-up evaluation 2 weeks after his surgery, the patient's diarrhea had resolved entirely.

Mesh erosion following laparoscopic hernia repair has been reported in a number of case reports [1-9]. While there have been reports of mesh erosion into the colon [1-3], colovesical [6] and enterocutaneous fistulae [7, 9], enterocolic fistula from mesh erosion appears to be extremely rare, and there is only one other described case in a patient with an enterocolo-cutaneous fistula described by Ott et al. [8]. In this patient's case, the likely etiology of diarrhea was enterocolic fistula given the complete symptom resolution with surgical removal of the mesh and takedown of the fistula.

Funding There was no grant or financial support received for this publication. 


\section{Compliance with Ethical Standards}

Conflict of interest All authors declare that they have no conflicts of interest.

Informed consent This article does not contain any studies with human participants or animals performed by any of the authors.

\section{References}

1. Murphy JW, Misra DC, Silverglide B. Sigmoid colonic fistula secondary to perfix-plug, left inguinal hernia repair. Hernia. 2006;10:436-438.

2. Gandhi D, Marcin S, Xin Z, Asha B, Kaswala D, Zamir B. Chronic abdominal pain secondary to mesh erosion into cecum following incisional hernia repair: a case report and literature review. Ann Gastroenterol. 2011;24:321-324.

3. Celik A, Kutun S, Koçkar S, Mengi N, Ulucanlar H, Cetin A. Colonoscopic removal of inguinal hernia mesh: report of a case and literature review. J Laparoendosc Adv Surg Tech A. 2005;15:408-410.

4. Hume RH, Bour J. Mesh migration following laparoscopic inguinal hernia repair. J Laparoendosc Surg. 1996;6:333-335.
5. Al-Subaie S, Al-Haddad N, Al-Yaqout W, Al-Hajeri M, Claus C. A case of a colocutaneous fistula: A rare complication of mesh migration into the sigmoid colon after open tension-free hernia repair. Int J Surg Case Rep. 2015;14:26-29.

6. Yoshino Y, Sato J, Nakui M. A case of colovesical fistula developed after internal inguinal hernia repair with mesh plug method. Nihon Rinsho Geka Gakkai Zasshi J Jpn Surg Assoc. 2013;74:2008-2012.

7. Sistla SC, Reddy R, Dharanipraganda K, Jagdish S. Enterocutaneous fistula due to mesh fixation in the repair of lateral incisional hernia: a case report. Cases J. 2008;1:370.

8. Ott V, Groebli Y, Schneider R. Late intestinal fistula formation after incisional hernia using intraperitoneal mesh. Hernia. 2005;9:103-104.

9. Bostanci O, Idiz UO, Yazar M, Mihmanli M. A rare complication of composite dual mesh: migration and enterocutaneous fistula formation. Case Rep Surg. 2015;2015:293659.

Publisher's Note Springer Nature remains neutral with regard to jurisdictional claims in published maps and institutional affiliations. 